\title{
Relationship between ABO blood groups and selected pregnancy conditions and neonatal diseases
}

\author{
Izabela M. Cendal@), Barbara Krolak-Olejnik® \\ Department of Neonatology, Wroclaw Medical University, Wroclaw, Poland
}

\begin{abstract}
The influence of the blood group on the occurrence and severity of diseases has aroused the curiosity of scientists for many years. The ABO group system is the best known and described blood group system. It is also the only system whose antibodies are constantly present in the blood serum. The most common blood type in Poland, according to data provided by Honorary blood donation and blood therapy, is group $\mathrm{A} \mathrm{Rh}^{+}$(plus), while the least common is group $\mathrm{AB} \mathrm{Rh}^{-}$(minus).

In studies of pregnant women scientists discovered the influence of blood type in the development of preeclampsia, gestational diabetes, the risk of preterm labor, and even COVID-19 infection. The impact of the mothers' blood group also affects the birth weight of newborns, as well as the development of hemolytic disease of the newborn due to the heterospecificity of $\mathrm{AB} 0$. The influence of the blood group on the increased risk of developing certain diseases and complications of the neonatal period has also been proven.

Therefore, it seems important to study blood groups of pregnant women and newborns of different nationalities, correlate the results with available reports and use this knowledge in everyday clinical practice. This will help to increase the speed of detection of diseases in pregnancy and neonatal period. It will also facilitate the management of the patient depending on their blood group.
\end{abstract}

Key words: ABO blood group; COVID-19; neonatal disease; preeclampsia; pregnancy; gestational diabetes mellitus

Ginekologia Polska 2021; 92, 11: 818-821

\section{INTRODUCTION}

ABO blood groups are defined by the carbohydrate moieties on the extracellular surface of the membrane of the red blood cells. $A B 0$ antigens are also present on the surface of various human cells and tissues, such as epithelium, sensory neurons, thrombocytes, and vascular endothelium. The AB0 system was the first of the 33 currently identified blood group systems and is the most important to transfusion medicine when testing blood donors and recipients for $A B 0$ compliance. These tests are fundamental to the prevention of hemolytic reactions [1].

Currently, the issue of the influence of the blood group on the coagulation system, infectious diseases and cancer is being investigated. People with blood group 0 have about 25-30\% lower plasma factor VIII and von Willebrand factor levels compared to people with other blood groups. In addition, blood group 0 has been found to be associated with an increased incidence of infections with cholera, mumps and tuberculosis; type A blood group with an increased frequency of $P$. aeruginosa infection and blood group type $B$ with a higher risk of gonorrhea, tuberculosis, S. pneumoniae, E. coli and Salmonella infections. Also, blood group $A B$ is associated with an increased incidence of $E$. coli and Salmonella infections [2]. Other studies have shown a significant increase in the risk of stomach cancer in patients with blood group A compared to people with blood group 0 . It has also been suspected that people with blood group 0 may have a reduced risk of pancreatic cancer compared to non-0 groups. Data from other studies show that ABO blood group is also associated with the risk of developing skin, ovarian and lung cancer, while it has not been found to be associated with colorectal and breast cancer [1].

\section{COVID-19}

Due to the current epidemiological situation, more and more studies raise the issue of factors influencing the course of SARS-CoV-2 infection. Researchers also studied the effect of blood type in pregnancy on COVID-19 infection. In a study

\section{Corresponding author:}

Izabela M. Cendal

Department of Neonatology, Wroclaw Medical University, 213 Borowska St., 50-566 Wroclaw, Poland

e-mail: izabela.cendal@student.umed.wroc.pl 
conducted in September 2020, Ahmed et al. examined 86 pregnant women with a positive test for COVID-19 and 269 pregnant women with a negative result. Although the largest group of pregnant women in this study had blood type 0 , patients with blood type $\mathrm{A}$ had higher relative risk of contracting COVID-19 infection. Simultaneously, blood group 0 was associated with a lower risk of developing this infection. Considering that SARS-CoV-2 can replicate in epithelial cells that express A and B antigens in the lungs, researchers hypothesized that in people with blood type 0 who have both anti-A and anti-B antibodies this may pose a lower risk of infection [3].

In another study by Mirzaie et al. involving a group of 56 pregnant women diagnosed with COVID-19, the risk of developing COVID-19 was higher in examined patients with blood group $\mathrm{AB} \mathrm{Rh}^{+}$and $0 \mathrm{Rh}^{-}$, than in pregnant women with other blood groups [4]. The above studies suggest that the blood group may have an influence on the development of COVID-19 infection in pregnant women, but due to limited research groups in the examples provided, no clear conclusions can be made regarding the groups of increased risk or protective effect. For this reason, and in view of the continuing pandemic situation, similar research should be pursued. This may contribute to better protection of pregnant women against this infection in the future.

\section{PREECLAMPSIA}

One of the most serious conditions complicating pregnancy is preeclampsia. Many scientists have studied the relationship between $\mathrm{AB} 0$ blood group and preeclampsia, but their results are contradictory. For this reason, many scientists are still looking for conclusive results on the risk and predictive factors of this condition [5-8]. Two different types of preeclampsia are described based on the time of onset of the disease symptoms - early and late-onset preeclampsia. In a study conducted by Mahasub et al. on 690 pregnant women (including 230 women with preeclampsia and 460 control group), blood group 0 was linked to significantly decreased risk of late preeclampsia. Whereas in the case of early-onset preeclampsia, overweight/obesity and blood group 0 have been shown to be important independent factors associated with preeclampsia [5]. In 2016 Franchini et al. provided systematic literature review where they confirmed the effect of ABO blood group on the risk of developing preeclampsia. In particular, it has been proved that women with a non-0 blood group have a slightly increased risk of this condition compared to women with blood group 0 [6]. In another study by Phaloprakarn et al. study on 5320 pregnant women, the researchers found that both $A$ and $A B$ blood types were significantly associated with risk of preeclampsia [7]. However, in the study published by Uzundere et al. involving 668 pregnant women, AB0 blood group was not strongly associated with any hypertensive disease of pregnancy [8].

Inconclusive results of these studies may indicate a possible influence of the blood group on the development of preeclampsia. However, further research in this direction will be required to confirm this hypothesis.

\section{GESTATIONAL DIABETES MELLITUS (GDM)}

While few studies have investigated the relationship between blood type and GDM, most of the results were inconsistent. An example is a hypothesis that blood group $A B$ is a protective factor against GDM found in a systematic literature review presented by Franchini et al. [6]. This seems to be contradicted in the study involving 5320 patients by Phaloprakarn et al., in which the ABO blood group was not associated with an increased risk of GDM [7]. In another study by Sapanont et al. on 600 pregnant women it was shown that blood group 0 is an independent factor doubling the risk of GDM [9]. Due to limited sources, the relationship of the blood group to GDM cannot be clearly confirmed. However, further research may support or contradict the hypotheses described above. The results of subsequent analyses may contribute to the development of another factor influencing vigilance during screening of pregnant women.

\section{BIRTH WEIGHT}

Apart from its impact on the conditions of gestational period, the mother's blood group also influences a number of neonatal complications. Scientists are trying to find the correlation between the mother's blood groups and the birth weight of the newborn. A total of 2177 pregnant women was included in the Beyazit et al. study. Mothers with B blood group had significantly higher risk of having low birth weight babies [10]. Also, in the study by Gloria-Bottini et al. suggestion was made that in male neonates, the negative effect of smoking during pregnancy on birth weight is more visible in mothers with blood type $A$ than in those with other blood groups [12]. In another study by Kothari et al., on 200 newborns the birth weight of AB blood group infants turned out to be significantly lower than that of the others. This difference was particularly noticeable in comparison to group 0 (mean birth weight for $A B$ blood group was 2,62 kg and for 0 blood group 3,01 kg) [11]. These studies suggest that both the blood type of the mother and the newborn may influence birth weight. In addition, other factors influencing weight gain during pregnancy, such as smoking, may also result in different birth weight depending on the blood group.

\section{NEUTROPENIA AT BIRTH}

Hypertensive disease of pregnancy, such as preeclampsia are associated with neonatal neutropenia. About $50 \%$ of 
newborns born to mother with preeclampsia will develop neutropenia [13]. Based on a study by McMahon on a group of 3,981 newborns, it was found that infants with blood type $A B$ are much more likely to develop postnatal neutropenia compared to children with other blood types [14]. On another study by McMahon, after the analysis of statistical data (1,525 newborns, 94 of whom had blood type $A B)$, it was shown that the probability of neutropenia is $14-89 \%$ higher for $A B$ blood group neonates [13].

\section{NEONATAL IMMUNE THROMBOCYTOPENIA}

\section{(NAIT)}

A study conducted in 2012 confirmed earlier reports that there is no correlation between the severity of NAIT and blood group AB0. However, it has been found that blood groups can influence the development of NAIT, although not as a primary trigger. Therefore, the authors conclude that the association of type $A B 0$ with the development of severe NAIT may result from a potential association with one or more genes encoding regulatory factors [15]. A more recent study from 2020 has shown that NAIT is more common in children of mothers with blood type A than among children of mothers with blood type 0 . Research has also shown that some newborns with blood type A or B have platelets which contain antigens that may be incompatible with maternal antigens. These findings emphasize the probability that children featuring higher $\mathrm{AB} 0$ antigen expression are at high risk of developing NAIT [16]. Further studies should be performed to find out whether the $\mathrm{ABO}$ antigens are related to increased risk of the development of NAIT.

\section{HEMOLYTIC DISEASE OF NEWBORNS}

The neonatal hemolytic disease comprises for nearly $20 \%$ of neonatal jaundice, whereas in $30 \%$ it is caused by AB0-Rhesus incompatibility. With the widespread prevention of Rh alloimmunisation, ABO hemolytic disease has become more common and occurs almost exclusively in blood group $A$ or $B$ infants born to blood group 0 mothers (AB0 heterospecifics) [17]. About $25 \%$ of pregnancies are heterospecific because the fetal red cells have agglutinogen A or $B$ absent in the mother, while maternal serum contains the appropriate agglutinin which is antagonistic to the fetal blood group. However, only one percent of all infants have clinical signs of hemolytic disease. The presence of lgG anti-A or anti-B hemolysin in maternal serum is essential for the diagnosis of $A B 0$ hemolytic disease [18].

Several studies have focused on maternal-fetal AB0 incompatibility and investigated whether the newborn's blood group $A$ or $B$ is a serious risk factor for hemolysis and/or severe hyperbilirubinemia. It often appeared that neonatal blood group B predisposed them to acute hemolysis and severe hyperbilirubinemia. Transfusions were statistically more frequent in the $\mathrm{B} 0$ group compared to the $\mathrm{A} 0$ group. In the Bel Hadj study from 2019 on a group of 98 newborns with symptomatic hemolytic disease, a significant decrease in hemoglobin levels was noticed in the B0 group compared to the $A 0$ group. Neonatal $A B 0$ hemolytic disease with type B0 incompatibility was significantly associated with a higher hemolysis rate with no difference in the incidence of severe hyperbilirubinemia compared to AO incompatibility. Hemolysis and anemia were also less frequent in the $\mathrm{A} 0$ group. In neonatal $\mathrm{ABO}$ hemolytic disease, it has been shown that neonatal blood group A or B has no major effect on the intensity of neonatal jaundice, but the risk of hemolysis is higher in case of B0 incompatibility [17]. A similar study was conducted by Kaplan et al., on a group of 164 newborns. In this trial, significantly more infants with the B0 group developed hyperbilirubinemia in the first 24 hours than their A0 counterparts. The age at which the first total bilirubin above the $95^{\text {th }}$ percentile was recorded was earlier among the B0 infants. Similarly, there was a tendency to start phototherapy earlier in newborns with $\mathrm{B} 0$ group. The reasons for the markedly increased severity of jaundice in the B0 subgroup are not clear. Maternal factors, including varying levels of anti-A or anti-B immunoglobulin $\mathrm{G}$, can influence the degree of hemolysis, and changes in IgG distribution can affect macrophage phagocytosis red blood cells. The results of this study indicate that heterospecific newborns with blood group $\mathrm{B} 0$ are at a higher risk of hyperbilirubinemia than their $\mathrm{A} 0$ counterparts [19].

A 13-year study by Christensen et al., analyzed the results for 42529 newborns, with a control group consisting of 29729 newborns with blood type 0. Those with blood group A or $\mathrm{B}$, born to mothers with group 0 , exhibited higher levels of total bilirubin in the first 10 days after birth than those in control group. Moreover, newborns in group $B$ had slightly higher levels of total bilirubin than newborns in group $A$. It was found that newborns in group $A$ or $B$, born to mothers in group 0 , did not develop bilirubin levels exceeding $25 \mathrm{mg} / \mathrm{dL}$ more often, nor did they require more frequent admission to hospital for jaundice treatment than group 0 infants born to women in group 0 [20].

\section{NECROTIZING ENTEROCOLITIS (NEC)}

Infants with blood groups other than 0 have an increased likelihood of a severe course of NEC. Significantly increased risk of death due to NEC has been documented in infants with blood group $A B$ compared to all other blood groups. Intestinal resections in infants with NEC showed more intense staining of endothelial tissue in blood group $A$ than in $B$ or $A B$ group [13]. In a study by Thomson et al. carried out on a group of 276 newborns diagnosed with NEC, there was no statistically significant difference between the sex of newborns who died of NEC and those who survived, but newborns who died 
of NEC had accordingly lower birth weight, and newborns of lower gestational age with $A B$ blood were crucially more likely to die from NEC. A significant difference in survival in the group of $A B$ newborns results not only from the disproportionate frequency of their deaths (43\%), but also from the short survival time. Even compared to any other individual blood group, newborns with blood type $A B$ remained at a remarkably increased risk of NEC mortality, but the risk was greatest if they were born to a mother with blood type $A$ or $A B$. Researchers suggest that the presence of both $A$ and $B$ antigens in newborns from the $A B$ blood group increases the chance of an immune response and may explain the much worse prognosis in the NEC [21].

\section{CONCLUSIONS}

Based on the provided studies, it can be concluded that blood groups influence the occurrence of pregnancy complications and neonatal diseases and may affect their severity. Due to the detected correlations between the $A B 0$ group system and these conditions and complications, it is possible to increase the diagnostic vigilance in pregnant women and newborns with a specific blood group. It seems important that the influence on the birth weight depending on the blood group was proven - the lowest weight was presented by newborns born of mothers with blood group B. However, the influence of external factors significantly influences the birth weight of newborns, which results in the fact that in the case of nicotinism during pregnancy lower birth weight occurs in newborns whose mothers had blood type A.

Moreover, the analyzed literature shows that children with the $A B$ blood group have higher risk of developing neonatal diseases, such as NEC and neutropenia. Furthermore, neonatal conditions with a proven increased risk of disease due to blood type should be more closely monitored in clinical practice. For example, more rigorous monitoring should be implemented in newborns with blood group B born to mothers with blood group 0 due to the increased risk of hemolytic disease in newborns [17]. However, due to the limited number of publications about neonatal diseases, further research on this concern will be required.

\section{Conflict of interest}

All authors declare no conflict of interest.

\section{REFERENCES}

1. Franchini M, Liumbruno GM. ABO blood group: old dogma, new perspectives. Clin Chem Lab Med. 2013; 51(8): 1545-1553, doi: 10.1515/cclm2013-0168, indexed in Pubmed: 23648637.
2. Ewald DR, Sumner SCJ. Blood type biochemistry and human disease. Wiley Interdiscip Rev Syst Biol Med. 2016; 8(6): 517-535, doi: 10.1002/wsbm.1355, indexed in Pubmed: 27599872.

3. Ahmed I, Quinn L, Tan BK. COVID-19 and the ABO blood group in pregnancy: A tale of two multiethnic cities. Int J Lab Hematol. 2021; 43(1): e45-e47, doi: 10.1111/ijlh.13355, indexed in Pubmed: 32996710.

4. Mirzaie M, Shafie H, Heidarifar R, et al. Relationship between ABO blood group type and COVID-19 outcome in pregnant women. J Cell Mol Anesth. 2020; 5(4): 282-283, doi: 10.22037/jcma.v5i4.32025.

5. Mahasub N, Boriboonhirunsarn D. Relationship between $A B O$ blood groups and preeclampsia. Hypertens Pregnancy. 2020; 39(3): 348-353, doi: 10.1080/10641955.2020.1777298, indexed in Pubmed: 32525417.

6. Franchini M, Mengoli C, Lippi G. Relationship between ABO blood group and pregnancy complications: a systematic literature analysis. Blood Transfus. 2016; 14(5): 441-448, doi: 10.2450/2016.0313-15, indexed in Pubmed: 27177402.

7. Phaloprakarn C, Tangjitgamol S. Maternal ABO blood group and adverse pregnancy outcomes. J Perinatol. 2013; 33(2): 107-111, doi: 10.1038/jp.2012.73, indexed in Pubmed: 22678143.

8. Uzundere $\mathrm{O}$, Kaçar C, Andan C, et al. Are ABO blood groups and Rh factor risk ractors for hypertensive diseases of pregnancy? Istanbul Medical Journal. 2020; 21(1): 53-57, doi: 10.4274/imj.galenos.2019.59354.

9. Sapanont K, Sunsaneevithayakul P, Boriboonhirunsarn D. Relationship between $A B O$ blood group and gestational diabetes mellitus. J Matern Fetal Neonatal Med. 2021; 34(8): 1255-1259, doi: 10.1080/14767058.2019.1633299, indexed in Pubmed: 31204532.

10. Beyazıt F, Pek E, Güngör A, et al. Effect of maternal ABO blood type on birth weight and preeclampsia. International Journal of Reproduc tion, Contraception, Obstetrics and Gynecology. 2017; 6(6): 2164, doi: 10.18203/2320-1770.ijrcog20172308.

11. Kothari LK, Jain TP, Lall KB, et al. ABO blood groups and birth weight. J Pediatr. 1969; 75(3): 480-482, doi: 10.1016/s0022-3476(69)80276-3, indexed in Pubmed: 5804196.

12. Gloria-Bottini F, Cozzoli E, Neri A, et al. Effect of smoking and ABO blood groups on maternal age at child bearing and on birth weight. Eur J Obstet Gynecol Reprod Biol. 2011; 159(1): 83-86, doi: 10.1016/j. ejogrb.2011.07.026, indexed in Pubmed: 21839572.

13. McMahon KE, Habeeb O, Bautista GM, et al. The association between AB blood group and neonatal disease. J Neonatal Perinatal Med. 2019; 12(1): 81-86, doi: 10.3233/NPM-17115, indexed in Pubmed: 30347622

14. McMahon K, Muraskas J. The correlation between ABO blood group and neonatal disease severity. Pediatrics. 2017; 140(63), doi: 10.1542/peds.140.1 meetingabstract.63.

15. Ahlen MT, Husebekk A, Killie MK, et al. The development of severe neonatal alloimmune thrombocytopenia due to anti-HPA-1a antibodies is correlated to maternal ABO genotypes. Clin Dev Immunol. 2012; 2012: 156867, doi: 10.1155/2012/156867, indexed in Pubmed: 22110529.

16. Arneth B. Neonatal immune incompatibilities between newborn and mother. J Clin Med. 2020; 9(5), doi: 10.3390/jcm9051470, indexed in Pubmed: 32422924.

17. Bel Hadj I, Boukhris R, Khalsi F, et al. ABO hemolytic disease of newborn: Does newborn's blood group a risk factor? Tunis Med. 2019; 97(3): 455-460, indexed in Pubmed: 31729720.

18. Lucas GN. Neonatal jaundice due to $A B O$ incompatibility in Sri Lankan Indian J Pediatr. 1996;63(3): 381-384, doi: 10.1007/BF02751534, indexed in Pubmed: 10830015.

19. Kaplan M, Hammerman C, Vreman HJ, et al. Hemolysis and hyperbilirubinemia in antiglobulin positive, direct $A B O$ blood group heterospecific neonates. J Pediatr. 2010; 157(5): 772-777, doi: 10.1016/j. jpeds.2010.05.024, indexed in Pubmed: 20598320.

20. Christensen RD, Baer VL, MacQueen BC, et al. ABO hemolytic disease of the fetus and newborn: thirteen years of data after implementing a universal bilirubin screening and management program. J Perinatol. 2018; 38(5): 517-525, doi: 10.1038/s41372-018-0048-4, indexed in Pubmed: 29410540.

21. Thomson T, Habeeb O, Dechristopher PJ, et al. Decreased survival in necrotizing enterocolitis is significantly associated with neonatal and maternal blood group: the AB isoagglutinin hypothesis. J Perinatol. 2012; 32(8): 626-630, doi: 10.1038/jp.2011.150, indexed in Pubmed: 22094489. 